\title{
Influence of deformation process in material at multiple cracking and fragmentation of nanocoating
}

\author{
P.O. Maruschak ${ }^{\text {a,* }}$, S.V. Panin ${ }^{\text {b }}$, S.R. Ignatovich ${ }^{\text {c }}$, I.M. Zakiev ${ }^{\text {c }}$, I.V. Konovalenko a , I.V. Lytvynenko ${ }^{\text {, }}$, \\ V.P. Sergeev ${ }^{b}$ \\ a Ternopil Ivan Pul'uj National Technical University, Ternopil 46001, Ukraine \\ ${ }^{\mathrm{b}}$ Institute of Strength Physics and Materials Science SB RAS, Tomsk 434021, Russia \\ ${ }^{\mathrm{c}}$ National Aviation University, Kyiv 03680, Ukraine
}

\section{A R T I C L E I N F O}

\section{Article history:}

Available online 10 January 2012

\section{Keywords:}

Plastic deformation

Multiple crack

Crack-like defect

Nanocoating

Fragmentation

\begin{abstract}
A B S T R A C T
Strain localisation in the heat resistant nanocoated steel at the stage of cracks nucleation, coalescence, and fragmentation of the nanocoating is investigated experimentally. Regularities in the self-organisation of plastic yielding processes and their relation to the deformation relief geometry are established.
\end{abstract}

(c) 2011 Elsevier Ltd. All rights reserved.

\section{Introduction}

It is known that the effect of the main crack-like defects on the residual life of materials and structures can be assessed by the approaches of fracture mechanics [1,2]. However, the methodological base for predicting the residual strength of nanomaterials with multiple defects, which have a potential for coalescence, is practically absent $[3,4]$.

As a rule, deformation and fragmentation of a nanocoating applied on the plastic macrobase takes place upon activation of the relaxation processes, which cause the formation of the orderly or off-oriented relief in the form of surface roughness and corrugations. According to Panin and co-authors data, such a distribution of strains on the coating surface may have an orderly "staggered" nature [3].

Nowadays, based on the Balokhonov and Romanova investigations of the space and time distribution of the localised strain zones, the existence of strain waves and their relation to shear processes is established [5]. This causes a "migration" of microconcentrators in the material. Moreover, the material deforms without a break of its solidity by means of a mutual rotation of structural element conglomerates.

Investigations into this mechanism allowed plotting distribution charts of shear stress waves. The autowave mode of generation of such waves is achieved due to the redistribution of the macrodeformation energy [6]. The use of the multilevel approach

\footnotetext{
* Corresponding author.

E-mail address: Maruschak@tu.edu.te.ua (P.O. Maruschak).
}

of physical mesomechanics allowed revealing the main regularities in the development of autowave processes on boundaries of nonhomogeneous systems and finding out the conditions for the formation of brittle crack networks [7]. However, further research on physical and mechanical aspects of the rotational physical and mechanical model, which takes into account the fragmentation of a nanocoating, remains topical, as well as the quantitative analysis of the effects involved.

The effect of the deformation and failure mechanisms of mechanical system "heat resistant steel-zirconium nanocoating" is investigated in this paper.

\section{Research technique}

Specimens from steel $25 \mathrm{Kh} 1 \mathrm{M} 1 \mathrm{~F}$ with the applied zirconium nanocoating were loaded under cyclic tensioning on the STM-100 test setup at: frequency $f=1 \mathrm{~Hz}, \sigma_{\max }=500 \mathrm{MPa}, \sigma_{\min }=0.1 \sigma_{\max }$. After failure of specimens, the fractographic analysis of the coating condition at different relative strain values was performed, Fig. 1a. In order to reveal the main regularities in the formation of the geometrical structure of multiple cracking of the zirconium nanocoating (crack size, crack step within the section analysed) the digital identification approaches were used, Fig. 1b [8].

The relative strain of microsections of the material analysed was determined from formula:

$\varepsilon=\frac{\left(l_{k}-l_{0}\right)}{l_{0}}$

where $l_{0}$ is the initial coating length; 


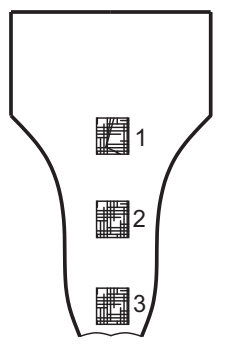

(a) Measurement of multiple cracking

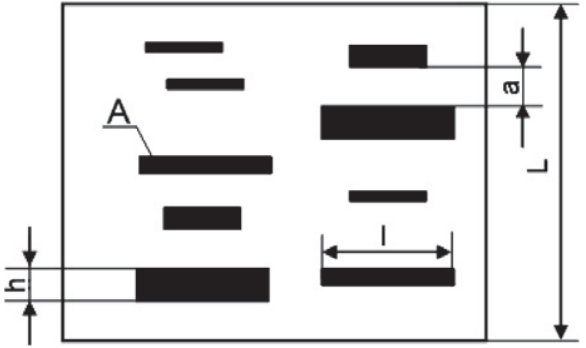

(b) Notations

Fig. 1. Scheme of deformed sections distribution and notations: $h$ is the crack thickness; $a$ is the distance between coating fragments; $l$ is the crack length; $A$ is the crack.

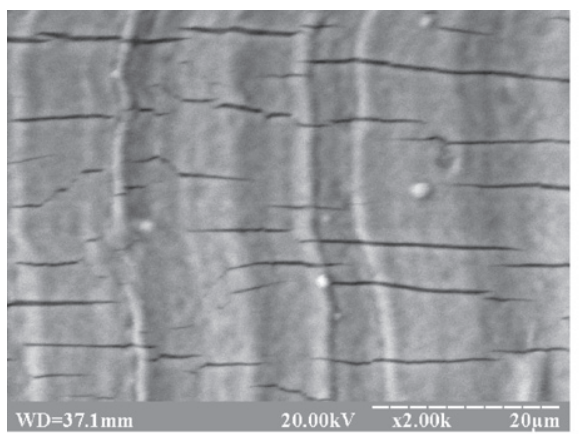

(a) $\varepsilon=5.9 \%$

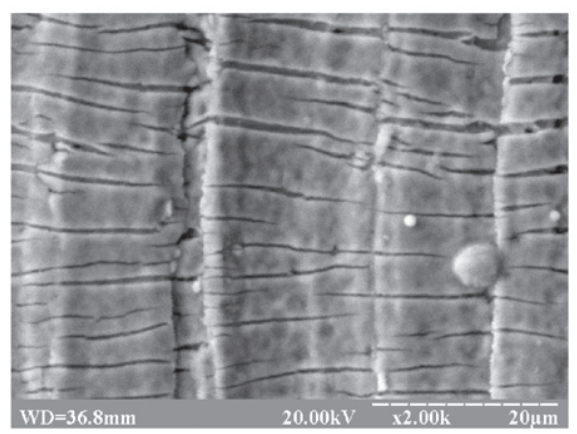

(c) $\varepsilon=15.3 \%$

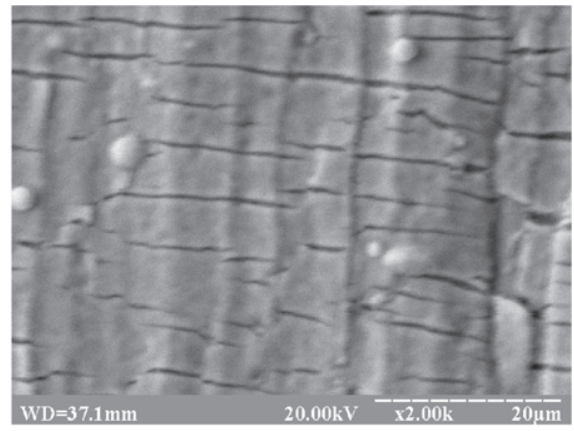

(b) $\varepsilon=7.8 \%$

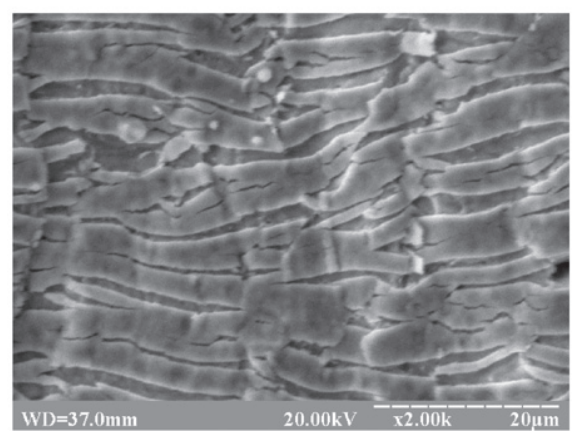

(d) $45.4 \%$

Fig. 2. Network of cracks in the deformed nanocoating at various values of relative strains.

It should be noted that surface deformation is defined by plastic yielding of the base and opening of multiple defects [9]:

$\varepsilon=\varepsilon^{i n t}+\sum_{i=1}^{p} \frac{\delta_{i}}{l_{k}}$

where $\varepsilon^{\text {int }}$ is the deformation of islands (fragments) of the material between cracks; $\delta_{i}$ is the opening of the $i$ th crack; $p$ is the number of cracks on the investigated length $l_{k}$.

The absolute strain of the coated material was determined as a difference between the final length of the cracked coating and the sum of shears of the cracked fragments from formula:

$l_{0}=l_{k}-\left(\sum_{i=1}^{p} \delta_{i}\right)$

where $l_{k}$ is the length of the cracked coating.

Based on the analysis of photo images, the structural and morphological data on cracks in the localised tensioning and shear zones were analysed. The spatial orientation of the crack network elements and their relation to the formation of meso- and macroscale fracture zones were analysed [6]. The "Micron-alpha" contactless 3D surface analyser was used for the analysis of the surface topography of specimens with cracks.

\section{Stage-like nature of nanocoating failure}

At the initial stage, cracks with predominantly horizontal orientation are nucleated, Fig. 2a. In addition, the "coalesced" cracks are detected, which are formed by means of coalescence of single defects, Fig. 2b. Plastic deformation of the specimen and localisation of strain in the specimen neck cause fragmentation of the coating in the direction perpendicular to that of loading, Fig. 2c. As a result, the surface layer acquires a micro-inhomogeneous structure, in which crack-like shears alternate with elements of the undeformed coating.

Depending on the density of the nucleated defects (the ratio between the length and width), their secondary fragmentation takes place, as well as a shift along the folds of the substrate material relief, Fig. $2 \mathrm{~d}$. The non-uniformity of shear strains in the coating is defined exactly by the deformation relief distribution and geometry.

Within the contraction zones, bands of the deformation relief are formed, which are distributed along the material surface, 


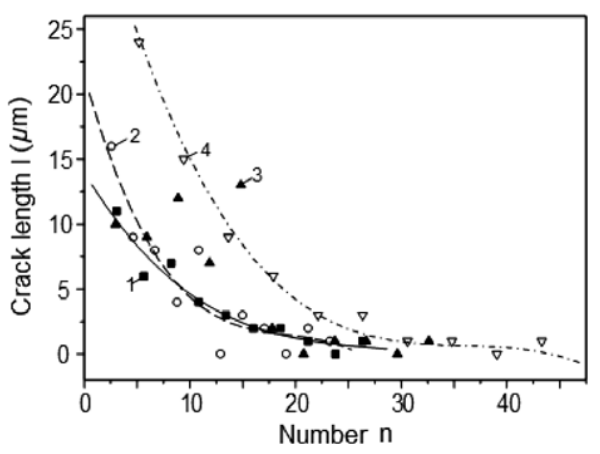

(a) Crack distribution on length

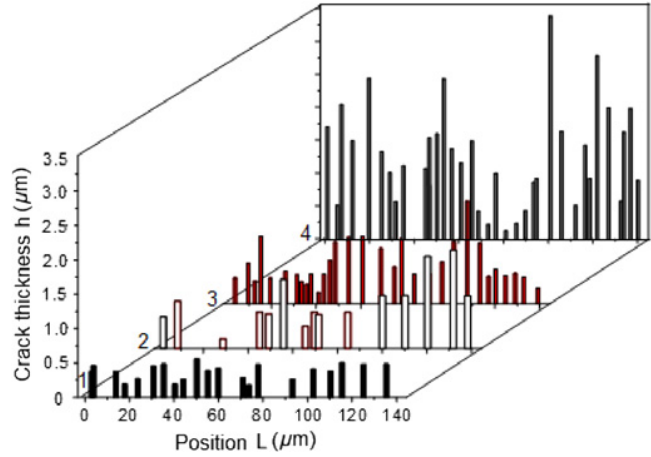

(b) Different relative strains

Fig. 3. Regularities in crack accumulation at various values of relative strain within a microsection: $\varepsilon=5.9 \%(1) ; 7.8 \%(2) ; 15.3 \%(3)$; $45.4 \%(4)$.

Fig. 2c. This leads to the occurrence of turning strains and microscale shears, which are the local relaxation zones [3]. An increase in the specimen necking activates shear processes in the material and causes the formation of a number of macroscale corrugations, Fig. 2d.

The results of the experimental assessment of multiple cracking based on the fractographic analysis data are given in Fig. 3.

Width distribution graphs of crack-like defects on the length analysed are plotted, Fig. 3a. A relationship is found between an increase in the amount of defects and an increase in their individual lengths, which allows making a conclusion about a significant nonuniformity of the individual crack growth process, Fig. 3b.

Table 1

Multiple cracking parameters of zirconium nanocoating.

\begin{tabular}{llll}
\hline No. & $\begin{array}{l}\text { Deformation, } \\
\varepsilon(\%)\end{array}$ & $\begin{array}{l}\text { The average width } \\
\text { of cracks }(\mu \mathrm{m})\end{array}$ & $\begin{array}{l}\text { Dispersion } \\
(\mu \mathrm{m})\end{array}$ \\
\hline 1 & 5.9 & 0.45 & 0.0015 \\
2 & 7.8 & 0.75 & 0.04 \\
3 & 15.3 & 0.67 & 0.13 \\
4 & 30.3 & 1.70 & 0.08 \\
5 & 45.4 & 1.45 & 0.22 \\
\hline
\end{tabular}

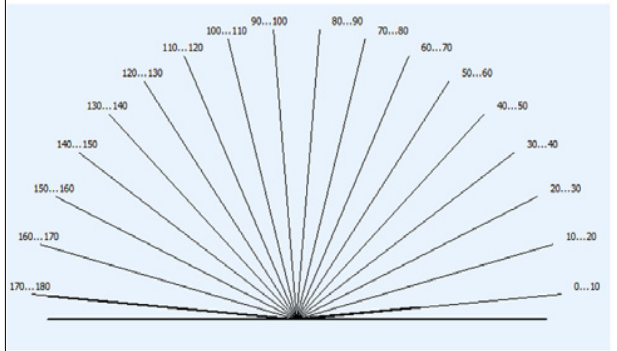

(a) $\varepsilon=5.9 \%$

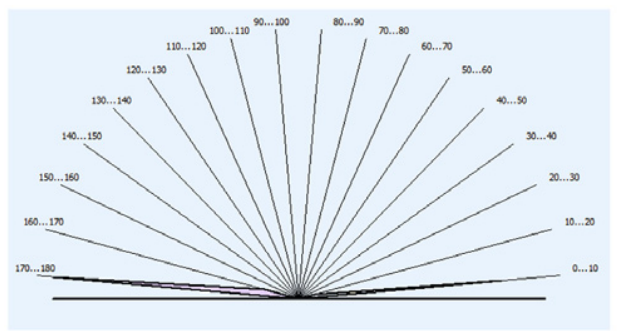

(c) $\varepsilon=15.3 \%$
We used the mathematical model of the cyclic random process with a zonal structure described in [11], which can be the given process of multiple cracking. The statistical methods for the assessment of the probability characteristics of such random processes were used.

The mathematical expectation was calculated from formula:

$\hat{m}_{h}(l)=\frac{1}{M} \sum_{n=1}^{M} h_{\omega}(l+L(l, n)), \quad l \in \mathbf{W}_{1}=\left[L_{1}, L_{2}\right)$,

where $L_{1} \neq 0$ in the general case, $M$ is the number of cycles during the process implementation, $h_{\omega}(L)$ is the crack width value (process implementation), $T(L, n)$ is the function of the rhythm of the multiple cracking process, $\mathbf{W}_{1}=\left[L_{1}, L_{2}\right)$ is the process definition area.

The dispersion was calculated from formula:

$\hat{d}_{h}(l)=\frac{1}{M-1} \cdot \sum_{n=1}^{M}\left[h_{\omega}(l+L(l, n))-\hat{m}_{h}(l+L(l, n))\right]^{2}, \quad l \in \mathbf{W}_{1}=\left[L_{1}, L_{2}\right)$.

The use of the modern mathematical apparatus allowed generalising the multiple cracking parameters of the nanocoating at different values of relative strain; the calculation results are compiled in Table 1.

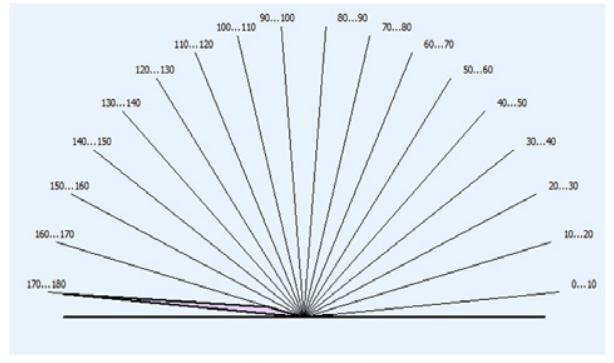

(b) $\varepsilon=7.8 \%$

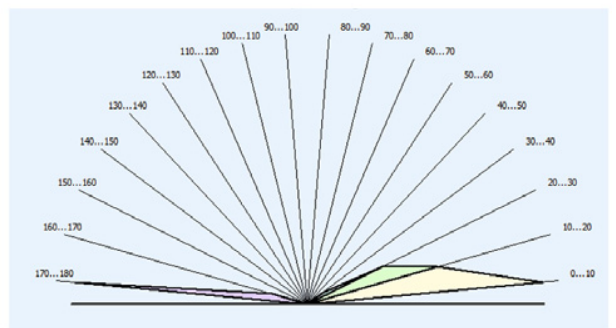

(d) $45.4 \%$

Fig. 4. Change in the orientation of crack-like microdefects in a deformed nanocoating at various values of relative strains. 


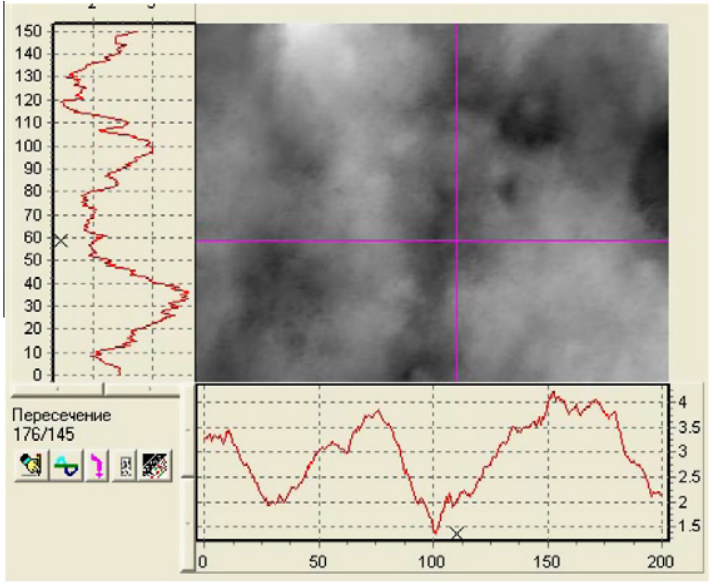

(a) Ref. $175 / 145$

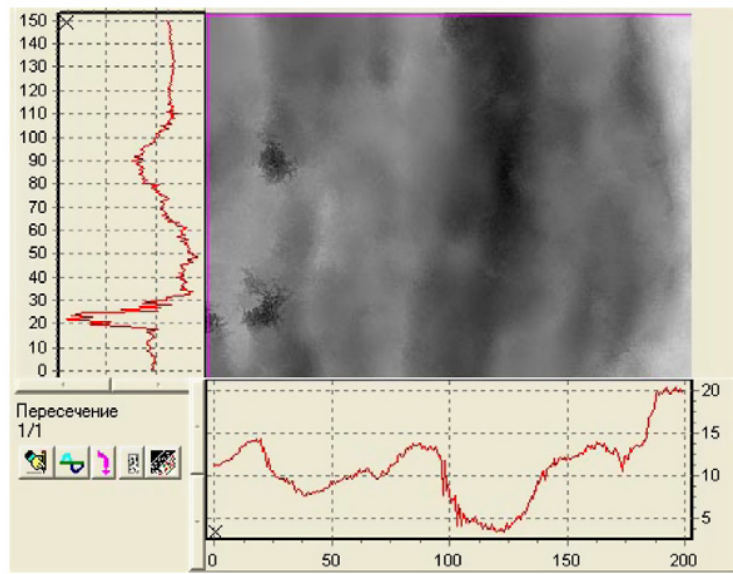

(b) Ref. 1/1

Fig. 5. Deformation relief detected on the surface of the neck of a specimen from steel 25Kh1M1F with a zirconium nanocoating in the specimen fracture zone.

While analysing the data from Table 1 , we can state that an increase in the strain level causes the formation of the orderly breakup-block structure of the coating and leads to its failure, which occurs in several stages. In addition, the combined deformation zones "contraction + shear" are the orderly geometrical singularity zones [10].

It should be noted that the initial array of cracks has a clear-cut orientation at an angle of $90^{\circ}$ to the loading axis, Fig. 4 . The coalescence of cracks increases the off-orientation of cracks by $9^{\circ}$. For relative strain $\varepsilon=15.3 \%$, a change in the character of deformation and relaxation of multiple sliding processes was observed. This is preconditioned by the alternation between the self-organisation and off-orientation processes in mesobands of plasticity.

At significant strains $(\varepsilon=45.4 \%)$, cracks are fully disoriented; during deformation, the processes of multiple fragmentation and shift of the coating mesofragments by $20^{\circ}$ take place.

Let us consider the profilogram of a fragment of the deformed coating surface in the vicinity of the specimen fracture zone. It is presented in the form of a 2D image supplemented by 2D cut-offs, Fig. 5.

It is found out that the shearing sections are formed due to fragmentation of the coating in proportion to the strain value. This evidences a certain stage-like nature of the fragmentation process.

The developed deformation bands can interact with each other forming the "interlaced" strain formations, Fig. 5a. This is characteristic of the bands with the inclined orientation, which are affected by shear stresses leading to local turning deformation processes that spread on the adjacent sections. It is the material sections involved in the shear and turning processes that stretch out protruding outside or create hollows, which form the deformation relief, Fig. $5 \mathrm{~b}$. It should be noted that regularly distributed fragments of the coating can be used as scale markers for the quantitative assessment of local deformation processes in the material [6].

In earlier works $[12,13]$ it was found out that further plastic yielding causes the formation of the regular deformation relief. The occurrence of such a relief testifies to the activation of compressive stresses in the direction normal to the deformation axis.

These processes cause the additional disorientation of the fragmented coating, however, the distance between bands is greater by one order of magnitude than the distance between the coating fragments. Surface irregularities acquire the shape of spirals oriented along the specimen axis. The presence of the nanocoating impedes a certain part of local shear strains, which are the sources
Table 2

Strain localisation in the heat resistant nanocoated steel at the stage of cracks nucleation, coalescence, and fragmentation of the nanocoating.

Stages of deformation and failure
microdefects (cracks) perpendicular to the
loading axis
Coalescence of microdefects and formation
of macrocracks due to the individual
growth of the available multiple defects and
nucleation of new ones. The coalescence or
brittle growth of cracks causes relaxation of
stresses within this section, which forces
the process of deformation to switch to
adjacent sections
Shear fragmentation of the coating into
fragments of different widths and
formation of the banded structure due to
deformation of the base material by means
of tensioning. Fragmentation of the coating
causes the activation of relaxation
processes and gradual formation of strain
localisation bands - corrugations
Localisation of deformation processes in the
base material leads to the occurrence of the
horizontal compression stresses. On local
sections of the specimen, the brittle and
plastic shift of failure processes in the
coating fragments takes place. In strain
localisation areas, the deformation relief is
formed. Shear processes are intensified in
the direction of the maximum shear
stresses. The formation of the orderly
fragmented structure due to the vortex
mesoturns of the base material and shift of
the coating fragments by the "shear + turn"
scheme take place. The mesoturns create
stable conglomerates of fragments with the
mutually agreeable turn

of local bending stresses that intensify the generation of local shear bands. Strain in these bands exceeds average values by several times, the process of yielding takes place by the scheme "tension + shear" on significant sections of the material, which alter- 


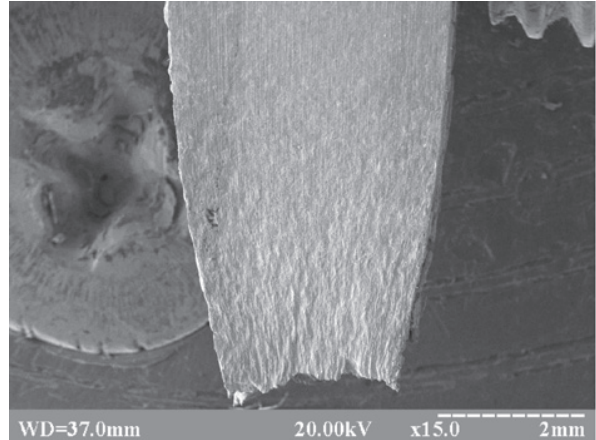

(a) $15 \mathrm{X}$

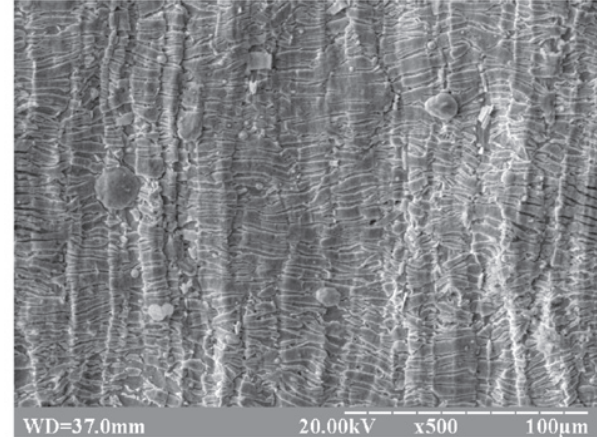

(b) $500 \mathrm{X}$

Fig. 6. Coating surface morphology generated under quasi-static tension.

nate with the plastic relaxation zones [3,4]. In addition, the earlier developed deformation structures remain intact and decorate the relief formations of a higher scale level. The size and number of such sections are connected with the initial structure of the material and the deformation regularities at the macrolevel [14].

The generalisation of the results obtained allowed drawing a scheme of the structural relaxation processes; it also permits identifying active mesodefects, determining the effect of their boundaries on the macroregularities in deformation and failure. A relationship between the nanocoating cracking morphology and the cyclic and quasistatic deformation processes in the material is established and given in Table. 2.

\subsection{Failure mechanisms of coated specimens}

The analysis of the main lines of research and the existing approaches to the problem of deformation of plastic solids with a hard coating shows that the ideas about the nonuniformity and localisation are actually characteristic of the physical theory of plasticity and strength [15]. In our case, the localisation of plastic strain manifests itself at all the scale and structural levels of the process in the form of sliding bands, fronts of plastic deformation, kink bands, space and time oscillations of plastic deformation, etc.

Numerous bands of plasticity located parallel to the loading axis are detected on the fractured specimen surface. These bands are formed in the specimen material and affect the separation boundaries of the coating leading to the initiation and hastening of mutual shears of the coating fragments [16]. They are an effective means of relaxation of local stresses in the material. Zones of the mesoplastic strain localisation are the packs of shears that occur along the sliding planes of the coating fragments. They realise themselves by the wave process scheme (switching waves), which consists in the successive alternation between the localised mesoscopic shears and turns in the material initiated by them. The formation of the quasiperiodic mesoband structures is linked to the relaxation of spatially oscillating stress mesoconcentrators in the coated material $[17,18]$.

Failure of the base material resulted from the completion of the mesofragmentation process and exhaustion of possibilities of this deformation mechanism as a relaxation process at the mesolevel. At this stage, a neck forms in the material, micropores coalesce and macrolocalisation takes place. Further failure of specimens is preconditioned by a disturbance of this self-coordination process and a growth of the noncompensated turning mode of deformation. A macrocrack, which appears in the material as the accommodative turning mode of deformation, propagates perpendicular to the axis of tension in this case. During strain localisation (formation of a neck), the coating separation is detected on individual sections of the separation boundary between the coating and the base.
The cohesive strength of the coating exceeds its adhesive strength, which leads to the coating separation during tensioning of the specimen. Obviously, the coating sustains tensile stresses while the coating layer bordering on the separation boundary sustains compressive stresses. Their relaxation takes place by separation of a part of the coating, Fig. $6 a$ and $b$.

Self-organisation of the deformation surface relief in the vicinity of the specimen fracture zone confirmed once again the validity of the wave theory of deformation connected with the processes of self-organisation in deformable mediums under quasistatic deformation $[19,20]$. Earlier, such waves were detected in a number of metallic materials, and the results on the mesoscopic strain localisation obtained in this work showed that the kinetics of deformation processes must be taken into account while interpreting multiple cracking, i.e. the formation of cracks, breaks, folds, fragmentation of the material and separation of the coating.

\section{Conclusions}

The results obtained prove that plastic deformation of the base material leads to the occurrence of multiple cracking and microfragmentation processes in the nanocoating, which have a tendency for self-organisation.

The nucleation and propagation of localised plasticity bands in steel specimens with the zirconium coating are investigated at the mesoscale level. The main regularities in changes in the shear and turn mechanisms of deformation of the fragmented nanocoating are obtained. It is found out that, during the localisation of plastic strains in mesovolumes, the voluminous structural elements can be formed, which contain the elements of the coating fragments shifted with a certain step.

The practical value of the results obtained is defined by the developed approaches to studying the effect of the multilevel structure of deformation and failure of nanocoatings and the development of the plastic yielding processes in the base material. The offered approaches have the universal character and can be used for the quantitative assessment of deformation processes in the break-up-block medium.

\section{References}

[1] V.V. Panasyuk, M.P. Savruk, A.P. Datsyshyn, A general method of solution of two-dimensional problems in the theory of cracks, Eng. Fract. Mech. 9 (1977) 481-497.

[2] M. Kachanov, On the problems interactions cracks and crack coalescence, Int. J. Fract. 120 (2003) 537-543.

[3] V.E. Panin, D.D. Moiseenko, A.L. Zhevlakov, P.V. Maksimov, Nucleation of mesoscopic localized deformation bands at the surface layer-substrate interface and their propagation in the bulk of a loaded solid, Tech. Phys. Lett. 34 (2008) 190-192. 
[4] V.I. Kushch, I. Sevostianov, L. Mishnaevsky Jr., Effect of crack orientation statistics on effective stiffness of mircocracked solid, Int. J. Solids Struct. 46 (2009) 1574-1588

[5] R.R. Balokhonov, V.A. Romanova, The effect of the irregular interface geometry in deformation and fracture of a steel substrate-boride coating composite, Int J. Plasticity 25 (11) (2009) 2025-2044.

[6] L. Mishnaevsky Jr., A simple method and program for the analysis of the microstructure-stiffness interrelations of composite materials, J. Compos. Mater. 41 (2007) 73-87.

[7] P. Yasniy, P. Maruschak, I. Konovalenko, V. Hlado, Local strains and mesoscopic mechanisms of crack growth in 25Kh1M1F steel, Strength, Fract. Complexity 6 (2010) 169-175

[8] P.V.Yasnii, P.O. Marushchak, I.V. Konovalenko, R.T. Bishchak, Computer analysis of surface cracks in structural elements, Mater. Sci. 44 (2008) 833-839.

[9] V.C. Li, H.C. Wu, Conditions for pseudo strain-hardening in fiber reinforced brittle matrix composites, J. Appl. Mech. Rev. 45 (1992) 390-398.

[10] V. Tamuzs, N. Romalis, V. Petrova, Fracture of Solids with Microdefects, Nova Science Publ., Inc., NY, 2000. 238p.

[11] I. Lytvynenko, S. Lupenko, Yu. Studena Statistical techniques of the heart signals processing based on of their model as a cyclic process of random band temporal structure, J. Ternopil State Technical University, vol. 11, 2006, pp. 189-200 (in Ukrainian).

[12] S.R. Ignatovich, Statistical nature of plastic deformation, Strength Mater. 28 (1996) 317-323.
[13] P. Yasniy, V. Hlado, P. Maruschak, D. Baran, Evaluation of hardening of plastically deformed steels, in: Proc. of the 13th International Conference "Experimental Analysis of Nano and Engineering Materials and Structures", Alexandroupolis, Greece, July 1-6, 2007.

[14] J. Pokluda, P. Šandera, Micromechanisms of fracture and fatigue, in: A Multiscale Context, Springer, 2010, p. 293.

[15] H. Gleiter, Nanocrystalline materials, Progress Mater. Sci. 33 (4) (1989) 223315.

[16] Y. Lapusta, W. Wagner, Estimation of the effects of initially closed interfacial cracks on fiber-matrix stability in compression, Int. J. Fract. 119 (2003) 71-76

[17] Y. Lapusta, F. Labesse-Jied, A. Samborskaya, W. Wagner, On the effects of interacting anisotropic fibers on the microbuckling in a composite, Int. J. Fract. 167 (2011) 103-110.

[18] P.V. Yasniy, P.O. Maruschak, S.V. Panin, P.S. Lyubutin, Strain stages and regularities of static fracture of $25 \mathrm{Cr} 1 \mathrm{Mo} 1 \mathrm{~V}$ steel damaged by a network of thermal fatigue cracks, in: 13th Int. conf "Mesomechanics 2011", July 6-8, Vicenza, Italy, 2011.

[19] L. Mishnaevsky Jr., S. Schmauder, Damage evolution and heterogeneity of materials: model based on fuzzy set theory, Eng. Fract. Mech. 57 (1997) 625636.

[20] V.I. Kushch, S.V. Shmegera, L. Mishnaevsky Jr., Meso cell model of fiber reinforced composite: interface stress statistics and debonding paths, Int. J. Solids Struct. (45) (2008) 2758-2784. 